Show simple item record

\title{
Physiological traits associated with drought resistance in Andean and Mesoamerican genotypes of common bean (Phaseolus vulgaris L.)
}

\begin{tabular}{|c|c|c|}
\hline dc.contributor.author & Polania, Jose Arnulfo & \\
\hline dc.contributor.author & Rao, Idupulapati M. & \\
\hline dc.contributor.author & Cajiao, César & \\
\hline dc.contributor.author & Rivera, Mariela & \\
\hline dc.contributor.author & raatz, bodo & \\
\hline dc.contributor.author & Beebe, Stephen E. & \\
\hline dc.date.accessioned & 2016-04-08T21:46:16Z & \\
\hline dc.date.available & 2016-04-08T21:46:16Z & \\
\hline dc.date.issued & 2016-04-06 & \\
\hline dc.identifier.citation & $\begin{array}{l}\text { Polania, Jose; Rao, Idupulapati Madhusudana; Cajiao, Cesar; Rivera, Mariela; Raatz, Bodo; Beebe, Stephen. 2016. Physiological traits } \\
\text { associated with drought resistance in Andean and Mesoamerican genotypes of common bean (Phaseolus vulgaris L.) . Euphytica 210(1): 17-29. }\end{array}$ & en_US \\
\hline dc.identifier.issn & $0014-2336$ & \\
\hline dc.identifier.uri & http://hdl.handle.net/10568/72866 & \\
\hline dc.format.extent & $210(1): 17-29$ & en_US \\
\hline dc.language.iso & en & en_US \\
\hline dc.publisher & Crossmark & en_US \\
\hline dc.source & Euphytica & en_US \\
\hline dc.subject & PHASEOLUS VULGARIS & en_US \\
\hline dc.subject & ABIOTIC STRESS & en_US \\
\hline dc.subject & WATER STRESS & en_US \\
\hline dc.subject & HARVEST INDEX & en_US \\
\hline dc.subject & COMMON BEANS & en_US \\
\hline dc.subject & BIOMASS & en_US \\
\hline dc.subject & ESTRÉS ABIÓTICO & en_US \\
\hline dc.subject & ESTRÉS HÍDRICO & en_US \\
\hline dc.subject & ÍNDICE DE COSECHA & en_US \\
\hline dc.subject & FRIJOL & en_US \\
\hline dc.subject & BIOMASA & en_US \\
\hline dc.title & Physiological traits associated with drought resistance in Andean and Mesoamerican genotypes of common bean (Phaseolus vulgaris L.) & en_US \\
\hline dc.description.version & Peer Review & en_US \\
\hline dc.type & Journal Article & en_US \\
\hline cg.subject.ciat & BEANS & en_US \\
\hline cg.identifier.status & Open Access & en_US \\
\hline cg.contributor.affiliation & International Center for Tropical Agriculture & en_US \\
\hline cg.identifier.doi & https://dx.doi.org/10.1007/s10681-016-1691-5 & en_US \\
\hline cg.isijournal & ISI Journal & en_US \\
\hline cg.contributor.crp & GRAIN LEGUMES & \\
\hline atmire.orcid.id & $0000-0002-8381-9358$ & \\
\hline
\end{tabular}




\title{
Files in this item
}

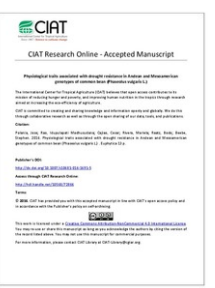

\author{
Name: \\ Physiological traits associated ... \\ Size: \\ 422.9Kb \\ Format: \\ PDF \\ Description: \\ Author's Version
}

View/Open

\section{This item appears in the following Collection(s)}

- CIAT Articles in Journals [1935]

- CIAT Agrobiodiversity [222]

Show simple item record

Show Statistical Information 